REDES- Revista hispana para el análisis de redes sociales

Vol.13,\#10, Diciembre 2007

http: // revista-redes.rediris.es

\title{
Redes sociales y mercado de trabajo
}

Livia García Faroldi - Universidad de Málaga ${ }^{1}$

\section{Reseña}

Requena Santos, F. (1991). Redes sociales y mercado de trabajo. Elementos para una teoría del capital relacional. Madrid: Centro de Investigaciones Sociológicas, Colección Monografías, № 119.

En 1973, Max Granovetter formula su teoría de la fuerza de los lazos débiles, reinvindicando que los analistas de redes tengan en cuenta la importancia de este tipo de vínculos para el individuo. El autor define la "fuerza" de un vínculo como una combinación (probablemente lineal) del tiempo, la intimidad (entendiendo por tal la confianza mutua), la intensidad emocional y los servicios recíprocos que caracterizan a dicho vínculo. Matiza Granovetter diciendo que cada aspecto es independiente de los otros tres aunque el conjunto está altamente interrelacionado.

Los vínculos débiles tienen una gran importancia en las redes egocéntricas. Granovetter sugiere que se puede dividir la red de ego en una parte formada por vínculos fuertes y débiles sin puente (entendiendo por puente el único camino entre dos puntos) y otra parte formada por vínculos débiles con puente. Es la segunda parte la que resulta de gran interés, porque los contactos de ego no están relacionados los unos con los otros y además están vinculados con individuos que no están conectados con ego. A través de estos canales se pueden alcanzar ideas, influencias e informaciones socialmente distantes de ego. En un estudio clásico (1974), el autor verificó su hipótesis de la relevancia de los vínculos débiles para la difusión de información. Estudió cómo una muestra de personas habían conseguido un nuevo trabajo el año anterior: sólo un 17\% respondió que lo había conseguido a través de amigos íntimos o familiares (lazos fuertes), por lo tanto, concluyó que los vínculos débiles sirvieron mayoritariamente como canales para obtener información para conseguir el nuevo empleo.

La teoría de la fuerza de los lazos débiles también tiene implicaciones profundas para las grandes estructuras, no sólo para los individuos (Granovetter, 1982). Los lazos débiles son vitales para la integración de un individuo en la sociedad

\footnotetext{
${ }^{1}$ Enviar correspondencia a: Igarcia@uma.es
} 
moderna, ya que la creciente especialización e interdepedencia en el trabajo genera una gran variedad de relaciones en las que un individuo conoce sólo un pequeño segmento de la personalidad del otro. Desde el punto de vista macrosociológico, su teoría señala que los sistemas sociales que carecen de lazos débiles estarán fragmentados y serán inconsistentes. Como consecuencia de ello, las nuevas ideas se difundirán lentamente y los subgrupos separados por cuestión de raza, etnia u otras características tendrán dificultades para convivir.

Las reflexiones de Granovetter sobre la importancia de los vínculos débiles como puentes locales para difundir información fueron empleadas por Burt (2001) para profundizar en el estudio de estas posiciones y las implicaciones que tienen en la creación de capital social. Frente al argumento de que el capital social es creado por una red de elementos fuertemente interconectados (closure argument, defendido, entre otros, por Coleman), Burt defiende que se crea a través de una red en la que las personas pueden ser intermediarias, es decir, actuar como puentes entre vacíos existentes en la estructura, conectando segmentos que en otro caso estarían aislados. El capital social se crea gracias a que el individuo participa en y controla la difusión de la información. El autor desarrolla su teoría de los agujeros estructurales (structural holes argument): los vacíos en la estructura social crean una ventaja competitiva para los individuos cuyas relaciones cruzan estos agujeros. Los miembros de cada lado del vacío estructural circulan por diferentes flujos de información $y$, por ello, las personas que actúan como puentes tienen la oportunidad de comunicar ambos flujos de información y controlar los proyectos que realicen juntos personas de los dos grupos. La existencia de un agujero estructural entre dos grupos no significa que los miembros de dichos grupos no sean conscientes de la existencia del otro, sino que están centrados en sus propias actividades de tal forma que no atienden las actividades de las personas del otro grupo.

Ocupar una posición de puente provee al individuo de varias ventajas con respecto al acceso a la información: llega a un mayor volumen de información porque contacta con más gente indirectamente; además, la diversidad de contactos entre varios grupos permite que conozca información menos redundante que la de otros individuos en otras posiciones, desempeñando el rol de líderes de opinión porque son los responsables de la difusión de nuevas ideas y comportamientos; por último, ocupar esta posición aumenta la probabilidad de ser un candidato para ser incluido en nuevas oportunidades que aparezcan en el mercado. 
La teoría de la fuerza de los lazos débiles ha tenido un gran éxito, no sólo en el ámbito del análisis de redes, sino en el mundo empresarial. Molina (2001) destaca cómo las consultoras que se encargan de recolocar a sus candidatos han incorporado estos conceptos a sus programas de formación e insisten en que los sujetos dediquen más tiempo a movilizar a sus contactos que a contestar anuncios de periódicos, pues el mayor porcentaje de recolocaciones se realiza mediante canales personales. Pese a la influencia de esta hipótesis en el análisis de redes, investigaciones posteriores han relativizado la importancia de los lazos débiles para lograr ventajas ocupacionales. Estos vínculos tienen un mayor protagonismo en el caso de las personas que pertenecen a un estatus elevado. El libro que nos ocupa, Redes sociales y mercado de trabajo, es precisamente el resultado de una investigación sobre cómo infuyen los lazos, fuertes y débiles, en el acceso al mercado de trabajo, y los datos muestran la enorme importancia de las relaciones más íntimas y los contactos directos.

Félix Requena Santos ha sido uno de los pioneros del análisis de redes en España. Su tesis doctoral fue una de las primeras en lograr una difusión amplia en el mundo de la investigación social española, consiguiendo el Premio Nacional de Trabajo y Seguridad Social del Ministerio de Trabajo y la publicación por parte del Centro de Investigaciones Sociológicas ( $\mathrm{CIS}$ ) en la monografía que nos ocupa. Su trayectoria posterior en este campo le ha valido la publicación de otros dos libros en el CIS sobre análisis de redes: un cuaderno metodológico (Redes sociales y cuestionarios, 1996) y una compilación de textos clásicos del análisis de redes (Análisis de redes sociales, 2003). En breve publicará una nueva obra en el CIS en la que analiza las relaciones entre las redes y la sociedad civil.

El libro se estructura en cinco capítulos, cuatro de ellos teóricos y uno sobre los resultados de la investigación. En la parte teórica existe, además de un análisis histórico-económico del mercado de trabajo, una introducción al análisis de redes en que se ofrece una panorámica general sobre los principales conceptos que se usan en esta disciplina. Posteriormente, desarrolla un modelo teórico en el que las redes sociales son un componente relevante para explicar cómo se busca y se consigue un empleo. Dado lo novedoso del tema en el contexto español, el autor se refiere con mayor frecuencia a estudios realizados en otros países que a investigaciones nacionales, aunque menciona la Encuesta de Población Activa de 1987 y un estudio de Sáez Fernández (1981) desarrollado en la segunda mitad de los setenta. 
En el momento en que se realizó la investigación (1989), el mercado de trabajo español vivía un momento de recesión económica unido a un exceso de población dispuesta a tener un empleo, debido a que muchos jóvenes de la generación del baby boom se incorporaron en esos años al mercado laboral. Desde el punto de vista funcionalista, Parsons (1984) señala que el problema de la regulación de los procesos de asignación de las personas es ver que la persona "adecuada" encaje en los roles adecuados. El sistema social se ocupa de regular los procesos para asignar a los individuos adecuados a través de la familia, el sistema educativo y fundamentalmente el sistema de relaciones sociales que rodean al sujeto. Los mecanismos de asignación ocupacional varían según la fase de ciclo económico y ciclo demográfico. En las circunstancias coyunturales ya comentadas, es más fácil asignar posiciones sociales de forma particularista que universalista, es decir, a través de la familia y las redes de amistad.

El modelo teórico propuesto por Requena intenta exponer, analizar y explicitar los mecanismos usados para la integración en la estructura ocupacional en circunstancias económicas y demográficas adversas. Existe una dualidad en los mecanismos de acceso al mercado de trabajo. Tales mecanismos se ven fuertemente influenciados por el origen social de los individuos que intentan lograr la entrada en el mercado ocupacional. La dependencia del origen de la persona que accede al mercado de trabajo ya no sólo está mediatizada por el estatus ocupacional de su progenitor sino por los grupos y/o instituciones primarias a los que pertenece el aspirante. El estatus social del entorno en el que está inserto el sujeto influirá en su posición dentro de determinadas redes sociales y, a su vez, la ubicación concreta dentro de la red es la que hace que ésta sea rentable para el individuo.

Requena centra su atención en el joven que se incorpora al mercado de trabajo, que recorre un camino determinado desde las instituciones primarias a las laborales. Los dos caminos para acceder al empleo son los métodos formales (servicios públicos de empleo, agencias o empresas dedicadas a la selección de personal, anuncios en la prensa, convocatorias a través de los centros de enseñanza, bolsas de trabajo de los sindicatos, etcétera) y los métodos informales (referencias de empleados, referencias de amigos, conocidos, familiares, parientes, solicitudes en la puerta, etcétera), englobados bajo el término genérico de redes sociales porque para usar estas referencias es necesario que los que dan las referencias y el interesado pertenezcan a la misma red social. 
Aunque los desempleados usan simultáneamente varios canales de búsqueda de empleo, los métodos informales se perciben como más operativos que los formales. Sin embargo, hay que tener en cuenta que según dónde se busque el trabajo habrá que potenciar unos canales de búsqueda u otros, porque las condiciones en las pequeñas empresas son más paternalistas y relajadas y los trabajos se encuentran casi siempre a través de contactos personales. También depende del tipo de trabajo que se busque, ya que los trabajos secundarios (siguiendo la distinción de Piore, 1983) se encuentran más probablemente a través de amigos y los primarios a través de parientes y familiares, tal y como muestra un estudio de Osterman (1983). Ello se debe a que a las empresas primarias les interesa la estabilidad, por lo que los padres y parientes son mejor fuente de referencia que los amigos porque pueden ejercer algún tipo de control sobre el empleado. Este control permite, por un lado, a los empleadores hacer una cierta selección por medio de los empleados que dan las referencias $y$, por otro lado, a los solicitantes obtener mucha información sobre la empresa y las condiciones de trabajo.

En todos los mercados de trabajo existe discriminación, aunque dicha discriminación afecte a grupos diversos según los contextos históricos y nacionales. En 1989, momento en que Requena realiza su trabajo de campo, el número de personas de minorías étnicas en España era muy reducido, por lo que el autor se centra en la discriminación por sexo y, sobre todo, por edad. En el año en que se realizó la encuesta, la cifra de parados, según la EPA, era de casi el 17\% (algo menos del $13 \%$ para los varones y $25 \%$ para las mujeres), concentrándose las mayores tasas de desempleo en los más jóvenes: casi el 37\% en los jóvenes de 16 a 19 años, más del 32\% entre 20 a 24 años y más del $22 \%$ de 25 a 29 años, siempre con cifras sensiblemente superiores para las mujeres que para los hombres. Los modos más frecuentes de discriminación son negarse a emplear a mujeres, jóvenes y minorías en puestos para los que están cualificados, emplearlos con salarios más bajos, exigir mayores cualificaciones si están contratados con los mismos salarios que los demás o impedir el acceso a los puestos mejor pagados.

Siguiendo el modelo de Atkinson (1987), una empresa flexibe consta de un núcleo donde se encuentran los mejores empleos, los mejor remunerados y los de mayor estatus, empleos a cuyo cargo se encuentran aquellas actividades que la empresa considera de mayor importancia y tiene en más alta estima. Estos trabajdores suelen ser varones con bastante experiencia, trabajan a jornada completa, de forma permanente y ponen en funcionamiento una serie de cualidades que la empresa no puede conseguir fácilmente en el exterior. Por otro lado, están los 
trabajadores de la periferia, que están a cargo de las actividades más mecánicas y rutinarias, es probable que sean mujeres y jóvenes que trabajen a tiempo parcial, con contratos eventuales y durante periodos breves. Estos trabajadores ponen en funcionamiento una serie de cualidades y técnicas que la empresa puede conseguir fácilmente en el mercado de trabajo externo. Esta división de los empleos muestra la discriminación que sufren ciertos colectivos en el mercado de trabajo.

La importancia de las redes sociales en el mercado de trabajo se relaciona con los costes que genera acceder a un puesto. Estos se reducen a los costes de búsqueda, que están determinados por los gastos necesarios de tiempo (en el que se deja de percibir un salario), de transporte (desplazamientos hacia las diversas instituciones que pueden contratar) y, sobre todo, de información. Este último es tal vez el coste determinante, pues si se supiera a ciencia cierta a dónde hay que ir a buscar un empleo y cuándo, el coste de búsqueda sería mínimo. La utilidad de las redes sociales se muestra en que, a través de los contactos y relaciones que generan, se abaratan todos los costes de acceso y búsqueda de empleo, por lo que es la respuesta social más efectiva. No existe, por tanto, una "libertad" de la mano de obra en el mercado de trabajo, sino que el individuo se ve fuertemente condicionado por su red de relaciones informales.

La mayor o menor eficacia de los contactos personales varían en función del estatus y de las características étnicas del individuo. Osterman (1983) muestra que la mayoría de los jóvenes blancos estadounidenses entrevistados consiguieron su trabajo porque un amigo que trabajaba en la empresa les informó que había una vacante, o porque su padre, un pariente o un vecino conocían a alguien en un puesto relevante en una empresa. Según el autor, esta red de contactos predominaba completamente sobre otras fuentes de adquisición de trabajos de los jóvenes de raza blanca. En cambio, los individuos de minorías étnicas tenían que recurrir normalmente a las oficinas de empleo para encontrar mejores trabajos, pues sus contactos sólo les llevaban a empleos sin posibilidades de carrera. Así, a medida que desciende el estatus, o se trata de minorías, es mayor el uso que se hace de los servicios estatales de empleo.

La estructura del mercado de trabajo español tiene importantes implicaciones a la hora de explicar cómo se asignan los inviduos a los puestos. La gran mayoría de las empresas son de reducido tamaño, por lo que los puestos se definen mal en cuanto a las profesiones exigidas, impidiendo en muchos casos que la selección del personal se realice de forma adecuada. Todo ello produce pésimos ajustes entre oferta y demanda de empleo. Las deficiencias funcionales de la estructura oficial 
generan otra estructura, no oficial, que pueda satisfacer las necesidades de asignación ocupacional de forma algo más eficaz. Las redes son una alternativa funcional, pues reducen los costes de búsqueda y generan una serie de controles sociales que logran una mayor eficiencia del individuo en su puesto de trabajo. Los mecanismos de asignación particularistas que mantienen los canales informales a través de las redes sociales sólo podrán ser sustituidos por mecanismos oficiales universalistas cuando sean más efectivos que los primeros para asignar empleos. Las relaciones personales siguen siendo el cauce por excelencia a la hora de conectar ofertas y demandas de puestos de trabajo, aunque se vayan imponiendo también los servicios públicos de empleo dado que es obligatorio solicitar el seguro de desempleo a través de estas oficinas oficiales $y$, también, porque se han puesto en marcha políticas de empleo en las que se subvencionan los contratos a personas con ciertas características. Estos dos motivos hacen que la población desempleada, sea cual sea su método de búsqueda, se inscriba, además, en las oficinas de empleo de la Administración. Requena denuncia que la obligatoriedad de inscribirse en las oficinas de empleo sesga los resultados de la Encuesta de Población Activa de 1987, que pregunta “¿cómo se está buscando un empleo?", en vez de “¿cómo se ha encontrado el empleo que se tiene?", sobredimensionando así la importancia de la oficina de empleo de la administración e infravalorando las relaciones personales.

En Italia, Sabel (1985) ha estudiado cómo se emplea la amistad y el parentesco para buscar un trabajo. Los trabajadores del sur del país que se trasladan a las zonas industrializadas del norte usan estas redes, lo cual tiene ventajas para trabajadores y empresas. En países con fuerte inmigración, las redes también se forman en torno a la pertenencia a una comunidad determinada: en Norteamérica, por ejemplo, los italianos recurrían a sus paisanos, que son redes de amigos y familiares originarios del mismo territorio que informan sobre los puestos de trabajo, atienden a los recién llegados y ofrecen apoyo emocional a aquellos que encuentran dificultades.

Otro aspecto del modelo de Requena es el concepto de capital relacional. Un factor clave en la obtención de cualificaciones en el segmento primario y de un puesto de trabajo en él es, según Doeringer y Piore (1983), la "aceptabilidad social", que no se puede "comprar" pero se puede conseguir, invirtiendo en contactos y relaciones sociales con miembros ubicados en posiciones más clave en las empresas que proporcionan empleos primarios. Esta accesibilidad está condicionada por otras variables intermedias como la clase social y el tipo de socialización que se haya tenido (capital cultural). Uno de los fenómenos que ha hecho rentable invertir en 
relaciones es la menor utilidad que tiene hoy un título escolar en el mercado, dada la multiplicación de títulos existente. La inversión en educación sigue siendo racional pero el estudiante debe redefinir el reparto de su tiempo entre estudios y otras actividades. El capital relacional depende de las posiciones relativas en las que un individuo puede encontrarse, pues el ocupar una posición estratégica en una red de relaciones manifiesta la posibilidad de tener más o menos información sobre situaciones determinadas. El coste de acceso a estas informaciones estará determinado por la centralidad del individuo en una red de relaciones. Esta parte del trabajo de Requena sobre el capital relacional es, precisamente, la que desarrolla Burt en sus investigaciones sobre las posiciones estratégicas en las organizaciones.

El capítulo de resultados comienza con una descripción de la metodología. El trabajo de campo consistió en una muestra aleatoria de 625 personas residentes en Málaga capital entre 16 y 65 años. Después de una descripción de la estructura ocupacional, educativa y por renta de la muestra, el autor se centra en los canales de búsqueda y acceso al empleo, la parte que es más interesante para el analista de redes. A la pregunta: “¿de qué forma está buscando trabajo?", los parados, con - sin experiencia laboral anterior, podían contestar hasta tres respuestas, ordenándolas según la mayor a menor esperanza que el encuestado tuviera en la efectividad de la gestión. La categoría más mencionada es "a través de amigos" $(31,3 \%)$, respuesta correspondiente al método en el que se tienen puestas mayores esperanzas de encontrar empleo; incluso es mayor esta categoría que la de los propios familiares. El siguiente porcentaje es el de la oficina de empleo, lo que se debe a la obligatoriedad de este canal oficial $(27,3)$. Los canales informales, si se suman los amigos $(31,3)$ y los familiares $(26,3)$, son un $57,6 \%$ de las respuestas, es decir, en los cauces no oficiales es donde se concentran las mayores esperanzas.

Diferenciando a los encuestados en dos grupos edad, los jóvenes (de 16 a 30 años) y los adultos (de 31 a 65 años), se observa que ambos emplean los mismos procedimientos de búsqueda, siendo el principal las relaciones personales. Pero si se distingue entre familia y amigos, los jóvenes utilizan con mayor frecuencia los canales familiares mientras que los adultos utilizan más los canales de amistad. Requena explica este fenómero refiriéndose a la estructura de las relaciones sociales en cuanto a poder se refiere: el joven con los únicos individuos con los que se relaciona que tengan más poder e influencia que él son los parientes, mientras que los adultos han tenido mayores posibilidades de generar contactos con no 
familiares. Además, la mayor experiencia y conocimiento del mercado de trabajo inclina a los interesados hacia el uso de los canales de amistad mientras que, por el contrario, se reducen ligeramente los mecanismos institucionales o formales. Por otro lado, ante la pregunta de cómo se ha conseguido empleo, los canales oficiales representan alrededor de un tercio del total y los informales dos tercios, con una diferencia de cinco puntos porcentuales a favor de los canales informales entre los empleados jóvenes (que se incorporaron al mercado después de la crisis de 1973) frente a los adultos. Sin embargo, hay que resaltar la diferente importancia de las redes sociales cuando se tiene en cuenta el nivel educativo de la ocupación de que se trate: los canales informales disminuyen su importancia al aumentar el nivel educativo.

La última parte del libro se dedica a describir las redes empleadas por los encuestados para buscar y conseguir un empleo. El autor distingue entre las redes sociales subjetivas, compuesta por aquellos individuos a los que el sujeto cree que podría recurrir en caso de necesidad, y las redes sociales objetivas, aquellas formadas por las personas a las que realmente se les pide ayuda en caso de necesidad. A su vez, se pueden diferenciar, en el caso del acceso al mercado de trabajo, entre las que usan los sujetos que están buscando empleo y las que usan los que ya lo han encontrado.

En cuanto a las redes que realmente se usan, Requena se centra en las de salida (las que parten del sujeto que solicita algo a los otros miembros). Si se estudian los tipos de relación de los que buscan empleo y los niveles por los que tienen que atravesar hasta llegar a las personas de las que se requiere ayuda, en los individuos que buscan empleo solamente se han evidenciado tres niveles o personas intermedias. El primer nivel es el más íntimo y a los demás se tiene acceso a partir del primero. Sin embargo, muchas veces (un $86,9 \%$ ) solamente se agota un primer nivel, es decir, se acude a personas con las que se tiene un contacto inmediato, independientemente del tipo de relación que se tenga con ella. En un $11 \%$ de los casos se accede a un segundo nivel de la red y en un 2,2\% al tercer nivel. Entre los que ya han encontrado trabajo y manifestaban haber solicitado ayuda a personas concretas para encontrarlo, se evidencian cuatro niveles. El 90,2\% solicita ayuda al nivel más cercano, donde las relaciones más mencionadas son las familiares $(28 \%)$ y las de amistad $(42,1 \%)$; el $8,8 \%$ usa un segundo nivel (y se diversifican los lazos, incluyéndose los compañeros de trabajo) y sólo un $1 \%$ agota los cuatro niveles. Estos resultados muestran que la mayoría 
encontró la información para acceder a un puesto a través de lazos fuertes, no de vínculos débiles.

Es interesante destacar que la red de los jóvenes que solicitan ayuda en la consecución de empleo es mucho menos densa que la de los más adultos que solicitan esa misma ayuda para conseguir empleo. La diferencia se debe a que las redes aumentan en densidad con la edad del individuo y a que la mayor densidad de la red en los individuos más mayores evidencia una mayor comprensión y conocimiento de las relaciones operativas en el mercado de trabajo.

Si se diferencia por sexo entre los que buscan y los que encuentran empleo, se aprecia que las redes menos densas se dan entre los que buscan empleo y no existen diferencias por sexos, mientras que entre los que se han integrado la red es más densa y existen diferencias por sexo: los hombres presentan una red más densa, llegando a tres niveles, mientras que las mujeres se concentran en mayor medida en el primer nivel y no alcanzan el tercer nivel de relación. Se aprecia también cierta relación entre el ocio y el uso del tiempo libre en la generación de redes sociales: los entrevistados que emplean más tiempo en la asistencia a bares, cafeterías, etcétera, presentan redes más densas y distribuidas entre los diferentes niveles de relación que aquellos que no asisten con tanta frecuencia a estos lugares, que concentran más sus relaciones en el primer nivel.

Como ya se ha comentado, el trabajo de Requena se desarrolló en un momento difícil para incorporarse al mercado laboral, debido a la recesión económica y la gran oferta de mano de obra. Sin embargo, estudios posteriores de diversos autores españoles han incluido preguntas sobre la forma de buscar y encontrar empleo por los jóvenes a mediados de los noventa y en el año 2004, fechas en que las circunstancias económicas y demográficas eran diferentes a las de finales de los ochenta. Aunque no todos estos trabajos diferencian los canales familiares de los canales de amistad, la pauta es similar a todos ellos: los cauces informales (redes sociales) representan alrededor de la mitad de las respuestas de los encuestados tanto de aquellos que buscan un empleo como de aquellos que lo han encontrado. Las oficinas de empleo ocupan un lugar destacado entre los jóvenes que buscan (con cifras entre un quinto y un tercio de los entrevistados mencionando este canal, salvo en el año 2004 en que la cifra es sensiblemente menor), pero pocos mencionan este cauce oficial como el que les proporcionó el empleo, no superando el $6 \%$ en el mejor de los casos (Requena Santos, 2006). 
La lectura de Redes sociales y mercado de trabajo resulta, pese a haber transcurrido más de quince años desde su publicación, plenamente actual. Las reflexiones que realiza el autor sobre la segmentación del mercado de trabajo, la discriminación en la asignación de los puestos y la importancia de las redes sociales según el estatus social del individuo son un acicate para analizar cómo las redes sociales son empleadas por los inmigrantes que llegan a España para conseguir un empleo o cualquier otro tipo de ayuda. En esta dirección se están realizando hoy en día numerosas investigaciones. En esta propia revista, en el volumen 11 (diciembre de 2006), podemos encontrar la reseña de De Miguel del libro Las redes sociales de los inmigrantes extranjeros en España, en el que Aparicio y Tornos han estudiado la efectividad de la ayuda recibida para conseguir una vivienda y los resultados muestran la eficacia de los cauces informales frente a los oficiales. Como refiere Requena en su investigación, los mecanismos para asignar un empleo a una persona varían según la fase del ciclo económico y del ciclo demográfico. Las corrientes inmigratorias suponen un factor relevante que modifica la estructura del mercado de trabajo y resulta de interés comprobar en qué medida usan los inmigrantes los canales formales y los informales para acceder a un trabajo o para encontrar un mejor puesto una vez se está empleado. Los resultados de estos estudios servirán de guía para que las instituciones públicas diseñen las políticas más adecuadas para lograr la integración de este colectivo. Sería deseable, además, que estos estudios incluyan en sus generadores de nombres la posibilidad de mencionar vínculos débiles, ya que el papel de estos lazos es fundamental que para que en una sociedad compleja como la española los subgrupos no vivan separados y sin comunicación entre ellos, tal y como advierte Granovetter que puede ocurrir si se carece de lazos que sirvan de puentes entre ellos.

\section{Bibliografía}

Atkinson, J. (1987). "Flexibilidad de empleo en los mercados laborales". Zona Abierta, 42, 1-42.

Burt, R.S. (2001). "Structural Holes versus Network Closure as Social Capital", en Lin, N., Cook, K.S. y Burt, R.S. (eds.), Social Capital: Theory and Research; Piscataway, NJ : Aldine Transaction, 31-56.

De Miguel Luken, V. (2006). "Inmigración y redes personales de apoyo". REDES. Revista Hispana para el Análisis de Redes Sociales, 11, \#10. 
Doeringer, P.B.y Piore,M.J. (1983). “El paro y el "mercado dual de trabajo"', en Toharia, L. (comp.), Mercado de trabajo: Teorías y aplicaciones. Madrid: Alianza, 307-320.

Granovetter, M. (1973). "The Strength of Weak Ties". American Journal of Sociology, 78, 1360-1380.

Granovetter, M. (1974). Getting a Job. A Study of Contacts and Careers. Cambridge: Harvard University Pres.

Granovetter, M. (1982). "The Strength of Weak Ties. A network Theory Revisited". En Marsden, P.V. y Lin, N. (eds.), Social Structure and Network Analysis. Beverly Hills: Sage Publications, 105-130.

Molina, J.L. (2001). El análisis de redes sociales. Una introducción. Barcelona: Edicions Bellaterra.

Osterman, P. (1983). “La estructura del mercado de trabajo de los hombres jóvenes", en Piore, M.J. (comp.), Paro e inflación: perspectivas institucionales y estructurales. Madrid: Alianza, 246-260.

Parsons, T. (1984). El sistema social. Madrid: Alianza.

Piore, M.J. (comp.). Paro e inflación: perspectivas institucionales y estructurales. Madrid: Alianza.

Requena Santos, F. (1996). Redes sociales y cuestionarios. Madrid: CIS, Cuadernos Metodológicos, no 18.

Requena Santos, F. (2003). Análisis de redes sociales. Orígenes, teorías y aplicaciones. Madrid: CIS, Monografías, № 198.

Requena Santos, F. (2006). "Redes sociales, mercado de trabajo y migraciones". X Congreso de Inmigración: Globalización y movimientos transnacionales. Almería. 20-21-22 de abril 2006.

Sabel, C.F. (1985); Trabajo y política. División del trabajo en la industria. Madrid: Ministerio de Trabajo.

Sáez Fernández, F. (1981). “Movilidad laboral y servicios públicos de empleo en España". Papeles de Economía Española, 8, 270-281. 